'Programa de formación como especialista en Gastroenterología. Escuela de Postgrado. Facultad de Medicina. Universidad de Chile. Santiago, Chile.

2Departamento de Medicina Interna, Sección de Gastroenterología, Hospital Clínico Universidad de Chile. Santiago, Chile. ${ }^{3}$ Gastroenterología. Clínica Santa María. Santiago, Chile.

${ }^{4}$ Departamento de Radiología Intervencional. Hospital Clínico Universidad de Chile. Santiago, Chile.

${ }^{5}$ Radiología Intervencional. Clínica Santa María. Santiago, Chile.

Recibido el 8 de febrero de 2017, aceptado el 13 de octubre de 2017.

Correspondencia a: Jaime Poniachik Teller Dirección: Santos Dumont 999, Independencia. jaime_poniachik@yahoo.es

\section{Escleroterapia endoscópica asistida mediante balón oclusivo como tratamiento de varices gástricas cardiofúndicas de alto riesgo: Reporte de dos casos y revisión de la literatura}

\author{
DANIEL DA COSTA ${ }^{1}$, CRISTIÁN MONTENEGRO ${ }^{2,3}$, \\ PATRICIO PALAVECINO ${ }^{4}$, GERMÁN LOBOS ${ }^{5}$, TOMÁS CERMENATI ${ }^{4}$, \\ JAIME PONIACHIK ${ }^{2,3}$
}

Management of gastrointestinal bleeding caused by fundal varices is particularly difficult to manage. The options are: transjugular intrahepatic portosystemic shunt (TIPS), endoscopic injection of cyanoacrylate or balloon-occluded retrograde transvenous obliteration (BRTO). We report a 63 year-old male with a cirrhosis caused by hepatitis $C$ and a 66 year-old female with a cirrhosis caused by a non-alcoholic steatohepatitis. Both patients had a gastrointestinal bleeding caused by fundal varices and were treated with sclerotherapy with cyanoacrylate assisted with BRTO. Flow was interrupted in the gastro-renal shunt by a femoral access in both patients. The male patient had a new bleeding two months later and died. In the female patient an endosonography performed nine months after the procedure showed absence of remaining varices.

(Rev Med Chile 2017; 145: 1336-1341)

Key words: Balloon Occlusion; Esophageal and Gastric Varices; Gastrointestinal Hemorrhage; Sclerotherapy.

\footnotetext{
L
} a hipertensión portal por cirrosis hepática induce el desarrollo de colaterales porto-sistémicas (shunts). Aquellos que se desarrollan en la unión gastroesofágica producen varices cuya ruptura origina hemorragia digestiva alta (HDA), con pronóstico ominoso ${ }^{1}$.

La clasificación de Sarin de varices gástricas incluye a las varices gastroesofágicas que se continúan desde el esófago a la curvatura menor (tipo GOV 1). Estas son tratadas mediante ligadura a nivel esofágico. Las varices gastroesofágicas que se continúan hacia el fondo gástrico (tipo GOV
2) y las varices gástricas aisladas del fondo (tipo IGV 1) son denominadas en conjunto varices cardiofúndicas ${ }^{2}$. Estas pueden sangrar a presiones portales inferiores $(15 \pm 0,8 \mathrm{mmHg})$ a las varices esofágicas $(21 \pm 0,4 \mathrm{mmHg})$. Poseen un alto flujo de entrada a través de la vena esplénica, ya sea por las venas gástricas cortas, por la vena gástrica posterior (o gástrica derecha) o por la vena coronaria (o gástrica izquierda). Su flujo de salida es a través de shunt gastrorrenal o esplenorrenal, los cuales están presentes en $85 \%$ de los casos $^{3}$ (Figura 1). 


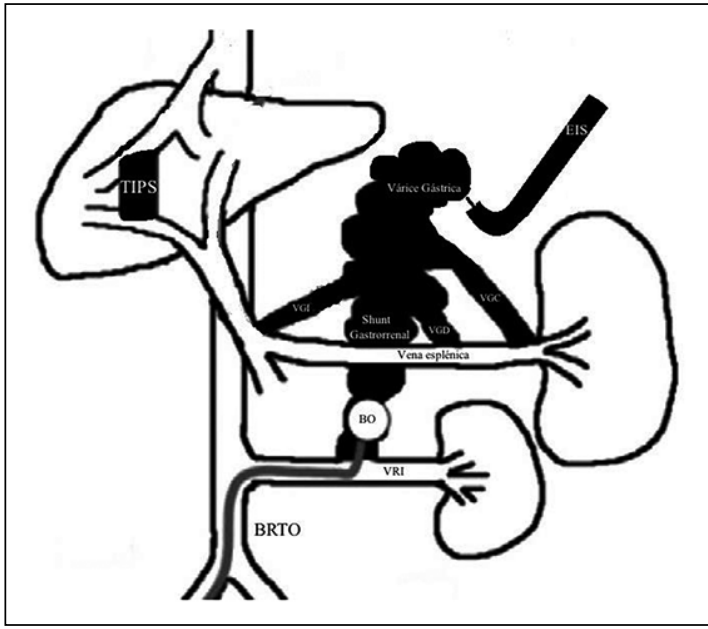

Figura 1. Las varices gástricas cardiofúndicas reciben afluentes desde la vena esplénica a través de las venas gástricas cortas (VGC), la vena gástrica derecha (VGD) y la vena gástrica izquierda (VGI). Drenan generalmente a través de shunt gastrorrenal por la vena renal izquierda (VRI) al sistema cava. Pueden tratarse reduciendo la presión portal mediante "shunt porto sistémico transhepático percutáneo" (TIPS), u obliterándolas inyectando cianoacrilato por vía endovascular a través de "obliteración retrógrada con balón" (BRTO) o inyectando endoscópicamente cianoacrilato (EIS) con asistencia de balón oclusivo (BO) por "escleroterapia endoscópica asistida mediante balón oclusivo" (BO-EIS).

Las varices gástricas cardiofúndicas son menos prevalentes y tienen menor riesgo de sangrado que las varices esofágicas, pero cuando sangran, su morbimortalidad es mayor ${ }^{2}$.

Sus opciones terapéuticas son la inyección con cianoacrilato, el shunt porto-sistémico transhepático percutáneo (TIPS) y la obliteración retrógrada con balón (BRTO) $)^{4-7}$.

Aunque la inyección con cianoacrilato es la terapia más utilizada, es frecuente el resangrado por obliteración incompleta y recurrencia. En estos casos, la reinyección es necesaria para lograr la obliteración completa. Sin embargo, el lumen de las varices gástricas ya obliteradas es pequeño, y la reinyección es dificultada por la presencia de polímeros de cianoacrilato inyectados previamente. En otros casos, el alto flujo y gran tamaño de las varices gástricas impiden el tratamiento endoscópico efectivo. Además, la inyección de cianoacrilato presenta complicaciones como embolia cerebral, pulmonar, portal, infarto esplénico y absceso retrogástrico ${ }^{8}$.
La obliteración retrógrada con balón (BRTO, por su sigla en inglés) es una técnica endovascular que se desarrolló para el tratamiento de las varices gástricas asociadas a shunt gastrorrenal o gastrofrénico. En esta, el esclerosante es inyectado dentro de la variz gástrica, mientras que el flujo de sangre variceal es detenido mediante balón de oclusión retrógrado. Su éxito en la obliteración de las varices gástricas es alto (87-100\%), con baja frecuencia de recaída $(0-10 \%)^{9-11}$. En un estudio comparativo con cianoacrilato, el resangrado fue más frecuente en pacientes tratados con cianoacrilato $(71 \%)$ versus aquellos con BRTO (15\%) $(\mathrm{p}<0,001)^{12}$.

Un trabajo de distribución aleatoria comparó TIPS y BRTO $^{13}$. No se demostró diferencias en mortalidad ni en control de hemorragia, sólo aparición de encefalopatía hepática en algunos casos de los pacientes tratados con TIPS $(\mathrm{p}=0,02)$.

En caso de no poder realizar BRTO, la obliteración percutánea transparietohepática (PTO) parece ser una alternativa equivalente ${ }^{14}$.

La escleroterapia endoscópica asistida mediante balón oclusivo (BO-EIS, por su sigla en inglés) es una técnica para tratar las varices gástricas que utiliza escleroterapia endoscópica después de bloquear su drenaje mediante balón endosvascular, generalmente ubicado en el shunt gastrorrenal. Comparado con BRTO, el volumen de esclerosante es significativamente menor, sin presentar desarrollo de varices esofágicas ${ }^{8}$ (Figura 1 y Tabla 1).

A continuación, se presentan dos casos clínicos de pacientes con varices gástricas de alto riesgo tratados mediante BO-EIS.

\section{Caso clínico}

\section{Caso 1}

Paciente de 63 años de sexo masculino, con antecedentes de cirrosis por virus hepatitis $\mathrm{C}$ y alcohol, Child C, MELD 14, encefalopatía hepática, ascitis y HDA recurrente por varices esofágicas y gástricas cardiofúndicas. Fue hospitalizado en Clínica Santa María en diciembre de 2015 por hematemesis y melena. Se trató mediante terlipresina. Se realizó endoscopia digestiva alta que mostró varices esofágicas pequeñas y varices gástricas cardiofúndicas grandes. Se realizó resonancia magnética que mostró varices perigástricas y de fondo gástrico, shunt gastrorrenal y esplenorrenal, 
Tabla 1. Opciones terapéuticas de varices gástricas cardiofúndicas

\begin{tabular}{|c|c|c|c|}
\hline & $\begin{array}{l}\text { Riesgo de resangrado } \\
\text { (Referencia) }\end{array}$ & Ventajas & Desventajas \\
\hline $\begin{array}{l}\text { Ciano- } \\
\text { acrilato }\end{array}$ & $\begin{array}{l}\text { Mayor que TIPS (6) } \\
\text { Mayor que BRTO (12) }\end{array}$ & - Lograr control local de la hemorragia & $\begin{array}{l}\text { - Falta de estandarización } \\
\text { - Riesgo embólico } \\
\text { - Riesgo de impacto en aguja } \\
\text { - Frecuente oclusión parcial }\end{array}$ \\
\hline TIPS & Equivalente a BRTO (13) & - Reducir ascitis & $\begin{array}{l}\text { - Producir encefalopatía hepática } \\
\text { - Complicaciones instalación } \\
\text { - Complicaciones cardiovasculares } \\
\text { - Trombosis }\end{array}$ \\
\hline BRTO & $\begin{array}{l}\text { Equivalente a TIPS (13) } \\
\text { Equivalente a BO-EIS (8) }\end{array}$ & $\begin{array}{l}\text { - Aumentar el flujo portal hepático } \\
\text { - Mejorar el MELD } \\
\text { - Oclusión definitiva de variz } \\
\text { - Sin riesgo de encefalopatía hepática } \\
\text { - Bajo riesgo embólico }\end{array}$ & $\begin{array}{l}\text { - Producir progresión de várices esofá- } \\
\text { gicas } \\
\text { - Producir ascitis } \\
\text { - Utilizar alto volumen de cianoacrilato } \\
\text { - Requerir de la presencia de shunt } \\
\text { gastrorrenal }\end{array}$ \\
\hline BO-EIS & Equivalente a BRTO (8) & $\begin{array}{l}\text { - No producir progresión de várices } \\
\text { esofágicas } \\
\text { - Se puede realizar en ausencia de } \\
\text { shunt gastrorrenal } \\
\text { - Presentar menor riesgo embólico que } \\
\text { BRTO } \\
\text { - Utilizar menor volumen de esclerosan- } \\
\text { te que BRTO }\end{array}$ & $\begin{array}{l}\text { - Requerir de radiología intervencional } \\
\text { y endoscopista }\end{array}$ \\
\hline
\end{tabular}

hepatocarcinoma y extensa trombosis de vena porta izquierda.

Ante la situación de paciente con HDA recurrente por varices esofágicas y ascitis se decidió no realizar BRTO y por encefalopatía hepática y trombosis portal no se instaló TIPS, por lo que se decidió realizar BO-EIS.

En sala de angiografía, bajo anestesia general, se puncionó la vena femoral común derecha con técnica de Seldinger. Se realizó cavografía y se canuló vena renal izquierda. Se evidenció shunt gastrorrenal. Se instaló balón Cudda y se infló excluyendo el shunt. Por endoscopía se procedió a inyectar 8 ampollas de cianoacrilato directo en las varices gástricas. Se utilizó dilución de cianoacrilato $0,5 \mathrm{cc}$ en $0,7 \mathrm{cc}$ de lipiodol. La cantidad de cianoactilato inyectada fue por criterio del endoscopista. Se comprobó que no hubo paso de material esclerosante al territorio sistémico o portal (Figura 2).

El paciente tuvo un nuevo episodio de HDA variceal a los dos meses, el cual fue tratado con inyección de 14 ampollas de cianoacrilato mediante nuevo procedimiento BO-EIS. Se decidió proporcionar el esfuerzo terapéutico y el paciente falleció.

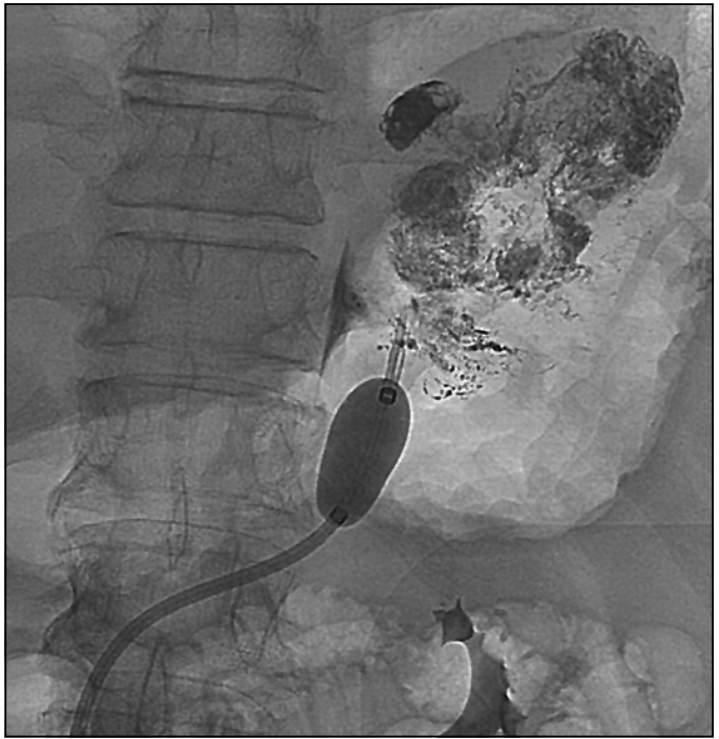

Figura 2. Por vena femoral común derecha se avanzó catéter hasta vena renal izquierda. Posterior a venografía que mostró shunt gastrorrenal, se infló balón Cudda excluyendo el shunt. Por endoscopía se procedió a inyectar 8 ampollas de cianoacrilato directo en las varices gástricas. En la figura, no se observa evidencia de paso de material esclerosante al territorio sistémico o portal. 


\section{Caso 2}

Paciente de 66 años de sexo femenino, con antecedentes de cirrosis por esteatohepatitis no alcohólica, Child A, MELD 12, ascitis, encefalopatía hepática y HDA recurrente por varices gástricas cardiofúndicas. Ingresó en forma electiva en enero de 2016 para BO-EIS.

En esta paciente se realizó acceso transparietohepático con acceso al sistema portal y a través de este al shunt anterógrado gastroesplénico, lo que permitió ocluir afluente de la variz gástrica. Por acceso femoral común derecho, a través de vena renal izquierda, se ocluyó el shunt gastrorrenal mediante balón Cudda. Así, con el circuito de las varices gástricas ocluido, por vía endoscópica, se inyectaron 7 ampollas de cianoacrilato directo sobre el paquete varicoso (Figura 3). Controlado por radioscopía, no se observó paso de material esclerosante al sistema portal ni sistémico.

La paciente evolucionó en forma favorable, dándose de alta al segundo día. Endosonografía realizada al noveno mes mostró ausencia de varices remanentes.

\section{Discusión}

Nuestro primer paciente presentaba HDA variceal recurrente, ascitis, encefalopatía hepática y hepatocarcinoma con invasión portal. Ante el fracaso de la inyección endoscópica con cianoacrilato, la imposibilidad de realizar TIPS por encefalopatía hepática y de realizar BRTO por ascitis y HDA recurrente por varices esofágicas se decidió realizar BO-EIS, con lo que se logró control de la HDA.

Nuestra segunda paciente también presentaba HDA recurrente por grandes varices gástricas cardiofúndicas, encefalopatía hepática y ascitis. Por tener un gran shunt gastrorrenal demostrado por angioTAC, se optó por realizar BO-EIS, inyectando 7 ampollas de cianoacrilato. La paciente ha estado sin HDA durante 12 meses.

Las distintas alternativas terapéuticas para el manejo de las varices cardiofúndicas presentan ventajas y desventajas, las que se resumen en la Tabla 1.

En términos de resangrado, TIPS y BRTO son superiores a cianoacrilato, siendo equivalentes entre ellos ${ }^{10,12}$.

La terapia mediante BRTO tiene la ventaja de aumentar el flujo hepático, por lo que se puede

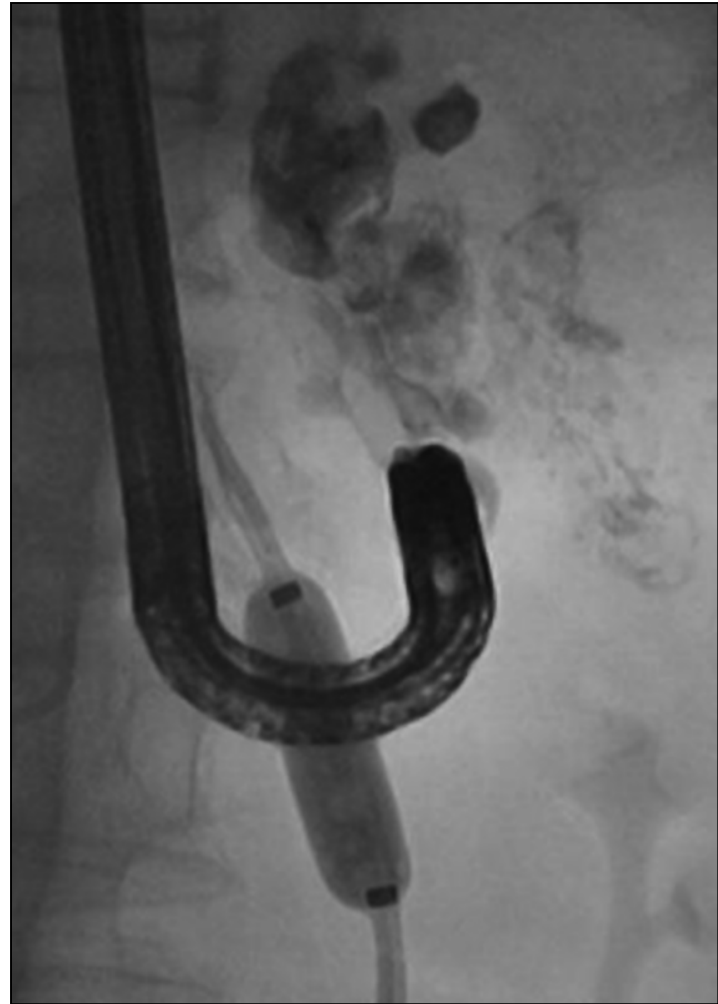

Figura 3. Por vía transparietohepática se accedió al sistema portal y se ocluyó shunt anterógrado gastroesplénico. Por acceso femoral se accedió al territorio sistémico y se ocluyó shunt retrógrado gastrorrenal. En la figura se observa inyecciónde cianoacrilato directo sobre las varices.

realizar en casos de disfunción hepática grave. El aumento del flujo hepático puede producir una mejoría del MELD de 3 a 6 puntos ${ }^{11}$. Además, BRTO produce oclusión definitiva de las varices, no presenta riesgo de encefalopatía hepática y posee mínimo riesgo embólico.

BRTO tiene las desventajas de poder aumentar la presión portal al ocluir el shunt, con desarrollo de varices esofágicas ( $25 \%$ de los casos) y aparición de ascitis o hidrotórax ${ }^{15}$. Esto se produce por obliteración permanente del shunt gastrorrenal secundario al alto volumen de cianoacrilato (promedio de $35 \mathrm{~mL})^{16}$. Además, no puede ser realizada en ausencia de shunt gastrorrenal (15\% de los pacientes).

La terapia mediante BO-EIS ha demostrado ser tan efectiva como BRTO en términos de resangrado. Su principal ventaja por sobre BRTO 
es no producir progresión de varices esofágicas ni aparición de ascitis, al no aumentar la presión portal. Esto es debido a la reducción del espacio para esclerosar y al menor uso de cianoacrilato (promedio $21 \mathrm{~mL}$ ), sin producir obliteración permanente del shunt ${ }^{8,17}$. Sus ventajas adicionales son que se puede realizar en ausencia de shunt gastrorrenal y presentar mínimo riesgo embólico.

Por lo tanto, al enfrentar a pacientes con HDA por varices gástricas cardiofúndicas, podemos tratarlos mediante inyección con cianoacrilato, TIPS, BRTO o BO-EIS. Por sus riesgos y menor eficacia en prevenir resangrado, sugerimos la inyección con cianoacrilato sólo ante la no disponibilidad de procedimientos endovasculares. En caso de disponibilidad de procedimientos endovasculares, se debe evaluar con imágenes. Si el paciente presenta además ascitis y ausencia de encefalopatía hepática, se puede ofrecer TIPS. En caso de shunt gastrorrenal, afluente único, ausencia de ascitis y ausencia de HDA esofágica recurrente, se puede indicar BRTO o BO-EIS. En caso de encefalopatía hepática, ascitis y HDA esófagica recurrente, BO-EIS parece ser la mejor opción, como fue en nuestros dos pacientes (Tabla 1).

En suma, BO-EIS parece ser equivalente a BRTO y TIPS en prevenir resangrado, siendo superior a inyectoterapia con cianoacrilato, con las ventajas de no producir progresión de varices esofágicas, no producir encefalopatía hepática, factibilidad en ausencia de shunt gastrorrenal, mínimo riesgo embólico y menor volumen de esclerosante. Se requieren estudios con mayor número de pacientes para confirmar éstas hipótesis.

\section{Referencias}

1. Candia R, Norero B, Pérez-Ayuso R, Fuster F, Zapata R, Fluxá F, et al. Manejo de varices gastroesofágicas: Revisión de la evidencia y consenso. Gastroenterol Latinoam 2011; 22 (1): 8-27.

2. Matsumoto A, Takimoto K. Gastric fundal varices: new aspects of non-surgical treatment in Japan. Nat Clin Pract Gastroenterol Hepatol 2006; 3 (1): 4-5.

3. Sarin SK, Lahoti D, Saxena SP, Murthy NS, Makwana UK. Prevalence, classification and natural history of gastric varices: a long-term follow-up study in 568 portal hypertension patients. Hepatology 1992; 16(6): 1343-9.

4. Lo GH, Lai KH, Cheng JS, Chen MH, Chiang HT. A prospective, randomized trial of butyl cyanoacrylate injection versus band ligation in the management of bleeding gastric varices. Hepatology 2001; 33(5): 10604.

5. Tan PC, Hou MC, Lin HC, Liu TT, Lee FY, Chang FY, Lee SD. A randomized trial of endoscopic treatment of acute gastric variceal hemorrhage: N-butyl- 2-cyanoacrylate injection versus band ligation. Hepatology 2006; 43 (4): 690-7.

6. Lo GH, Liang HL, Chen WC, Chen MH, Lai KH, Hsu $\mathrm{PI}$, et al. A prospective, randomized controlled trial of transjugular intrahepatic portosystemic shunt versus cyanoacrylate injection in the prevention of gastric variceal rebleeding. Endoscopy 2007; 39 (8): 679-85.

7. Mishra SR, Chander Sharma B, Kumar A, Sarin SK. Endoscopic cyanoacrylate injection versus beta-blocker for secondary prophylaxis of gastric variceal bleed: a randomised controlled trial. Gut 2010; 59: 729-35.

8. Shiba M, Higuchi K, Nakamura K, Itani A, Kuga T, Okazaki H, et al. Efficacy and safety of balloon-occluded endoscopic injection sclerotherapy as a prophylactic treatment for high-risk gastric fundal varices: a prospective, randomized, comparative clinical trial. Gastrointest Endosc 2002; 56 (4): 522-8.

9. Koito K, Namieno T, Nagakawa T, Morita K. Balloon-occluded retrograde transvenous obliteration for gastric varices with gastrorenal or gastrocaval collaterals. Am J Roentgenol 1996; 167 (5): 1317-20.

10. Chikamori F, Kuniyoshi N, Shibuya S, Takase Y. Shortterm hemodynamic effects of transjugular retrograde obliteration of gastric varices with gastrorenal shunt. Dig Surg 2000; 17 (4): 332-6.

11. Ninoi T, Nishida N, Kaminou T, Sakai Y, Kitayama T, Hamuro M, et al. Balloon-occluded retrograde transvenous obliteration of gastric varices with gastrorenal shunt: long-term follow-up in 78 patients. Am J Roentgenol 2005; 184 (4): 1340-6.

12. Hong CH, Kim HJ, Park JH, Park DI, Cho YK, Sohn CI, et al. Treatment of patients with gastric variceal hemorrhage: endoscopic N-butyl-2-cyanoacrylate injection versus balloon-occluded retrograde transvenous obliteration. J Gastroenterol Hepatol 2009; 24 (3): 372-8.

13. Choi YH, Yoon CJ, Park JH, Chung JW, Kwon JW, Choi GM. Balloon-occluded retrograde transvenous obliteration for gastric variceal bleeding: its feasibility compared with transjugular intrahepatic portosystemic shunt. Korean J Radiol 2003; 4 (2): 109-16.

14. Kwak HS, Han YM. Percutaneous transportal sclerotherapy with N-butyl-2-cyanoacrylate for gastric varices: technique and clinical efficacy. Korean J Radiol 2008; 9 (6): 526-33.

15. Fukuda T, Hirota S, Sugimura K. Long-term results of 
balloon-occluded retrograde transvenous obliteration for the treatment of gastric varices and hepatic encephalopathy. J Vasc Interv Radiol 2001; 12 (3): 327-36.

16. Akahane T, Iwasaki T, Kobayashi N, Tanabe N, Takahashi $\mathrm{N}$, Gama $\mathrm{H}$, et al. Changes in liver function parameters after occlusion of gastrorenal shunt with balloon-occluded retrograde transvenous obliteration. Am J Gastroenterol 1997; 92 (6): 1026-30.

17. Wu Q, Jiang H, Linghu E, Zhang L, Wang W, Zhang J, et al. BRTO assisted endoscopic Histoacryl injection in treating gastric varices with gastrorenal shunt. Minim Invasive Ther Allied Technol 2016; 25 (6): 337-44.

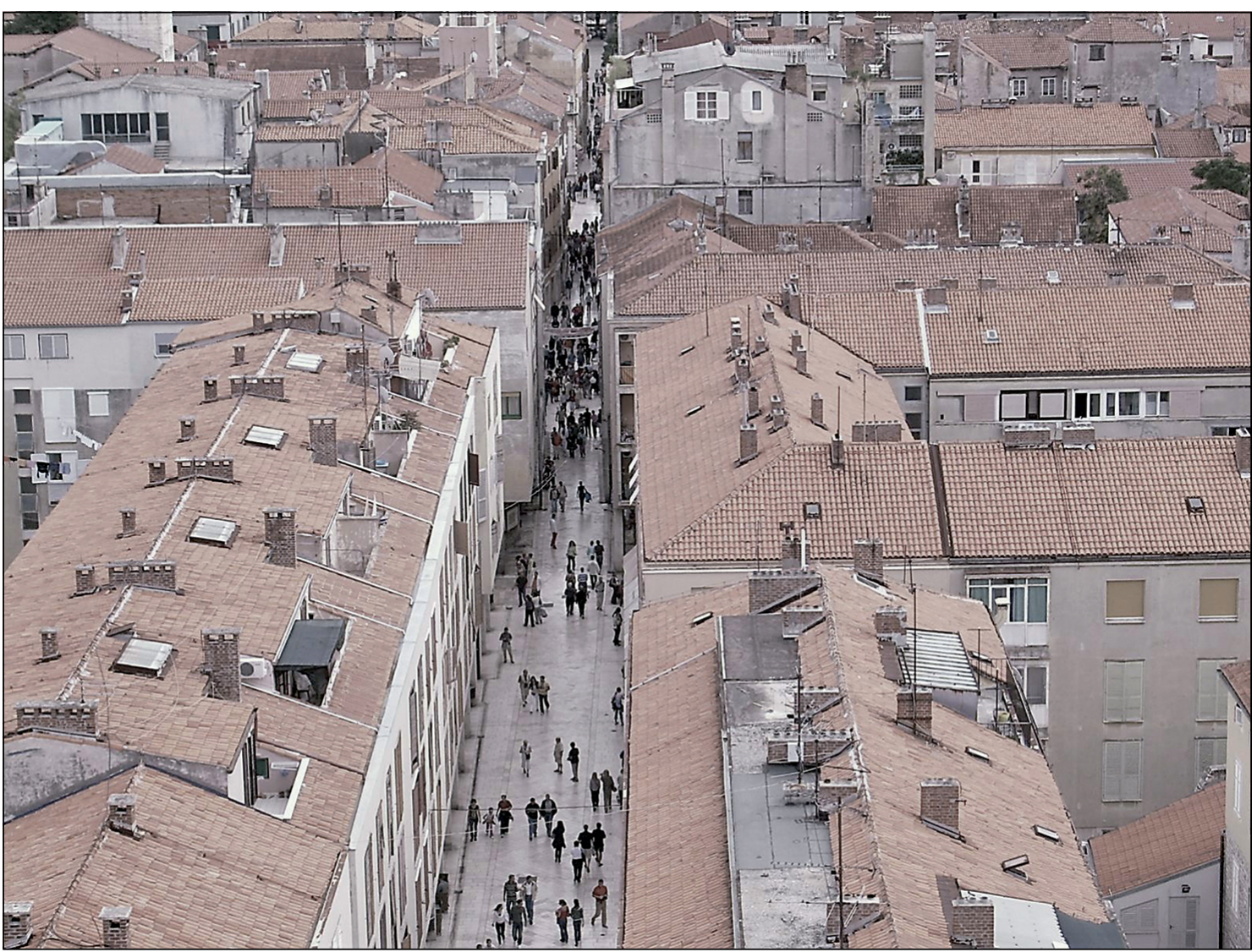

Zadar, Croacia. Dr. Joaquín Palma Heldt 